\title{
Association between HLA-DQ Gene Polymorphisms and HBV-Related Hepatocellular Carcinoma
}

\author{
Jingzhu Lv, ${ }^{1}$ Tao Xu, ${ }^{2,3,4}$ Zhongqing Qian, ${ }^{5,6}$ and Hongtao Wang ${ }^{5,6}$ \\ ${ }^{1}$ Department of Biochemistry and Molecular Biology, Bengbu Medical College, Bengbu 233003, China \\ ${ }^{2}$ Department of Clinical Laboratory, Bengbu Medical College, Bengbu, Anhui 233030, China \\ ${ }^{3}$ Department of Microbiology and Immunology, Medical School of Southeast University, Nanjing, Jiangsu 210009, China \\ ${ }^{4}$ Clinical Testing and Diagnose Experimental Center of Bengbu Medical College, Bengbu, Anhui 233030, China \\ ${ }^{5}$ Department of Immunology, Bengbu Medical College, Bengbu Anhui 233030, China \\ ${ }^{6}$ Anhui Key Laboratory of Infection and Immunity, Bengbu Medical College, Bengbu Anhui 233030, China
}

Correspondence should be addressed to Hongtao Wang; bb_wanghongtao@126.com

Received 27 March 2017; Revised 23 May 2017; Accepted 8 June 2017; Published 6 July 2017

Academic Editor: Tatsuya Toyokawa

Copyright (c) 2017 Jingzhu Lv et al. This is an open access article distributed under the Creative Commons Attribution License, which permits unrestricted use, distribution, and reproduction in any medium, provided the original work is properly cited.

\begin{abstract}
Hepatocellular carcinoma (HCC) is one of the most common causes of cancer-related mortality worldwide. Host gene variants may influence hepatitis B virus- (HBV-) related HCC. Human leukocyte antigens (HLA) play an important role in presenting virus antigens to immune cells that are responsible for the clearance of virus-infected cells and tumor cells. Previous studies have investigated the HLA-DQ (rs2856718 and rs9275572) polymorphisms that may be associated with the development of HBVrelated HCC. However, the results are controversial or inconclusive. Hence, we conducted a meta-analysis to derive a more precise estimation of the associations. A total of 6 articles were used to evaluate the effect of the two polymorphisms on the risk of HBV-related HCC. Odds ratios (ORs) and 95\% confidence intervals (95\% CIs) were calculated. We found that rs 2856718 and rs9275572 in HLA-DQ significantly decreased HBV-related HCC in total population, especially in Chinese, but not in Saudi Arabian. Further validation of our results in larger populations and different ethnicities are required.
\end{abstract}

\section{Introduction}

Primary liver cancer is one of the six most common cancers and the second largest cause of cancer deaths worldwide (746,000 cases or $9.1 \%$ of all cancer deaths), especially in undeveloped countries $[1,2]$. Approximately $75 \%$ of liver cancers occur in Asia, whereas China alone accounts for more than $50 \%$ of all cases [3]. Globally, the vast majority of histologic types of primary liver cancers (approximately 80\%) are hepatocellular carcinoma (HCC), a malignant tumor arising from hepatocytes, the liver's parenchymal cells [4]. It is estimated that $75 \%-85 \%$ of HCC patients are attributable to chronic infections with hepatitis B virus (HBV), especially in Asian populations and particularly in Chinese [5]. Besides HBV infection, other extrinsic factors, such as alcohol, smoking, physical inactivity, chemical exposure, and poor dietary habits, are also involved in developing HCC [6]. However, only a small fraction of infected patients can progress to HCC during their lifetime. So intrinsic factors, such as genetic mutations, may be vital for tumor development $[7,8]$. Furthermore, genetic epidemiology points out that genetic polymorphisms of genes involving in different processes of carcinogenesis may also play an important role to determine individual susceptibility to HCC and improve the prevention and treatment of this cancer [9-11].

Human leukocyte antigen (HLA) has been identified to be associated with regulating the immune response to HBV infection and clinical outcomes [12]. HLA-DQs are highly polymorphic especially in exon 2 , which codes for antigenbinding sites. The single-nucleotide polymorphism (SNP) rs2856718 locates in the intergenic region between HLADQA2 and HLA-DQB1. Hu et al.'s study showed that HLA-DQ rs2856718 significantly decreased the host HCC risk [13]. The SNP rs9275572 locates between HLA-DQA and HLA-DQB on 6p21.32. A recent genome-wide association study (GWAS) indicated that the HLA-DQ rs9275572 


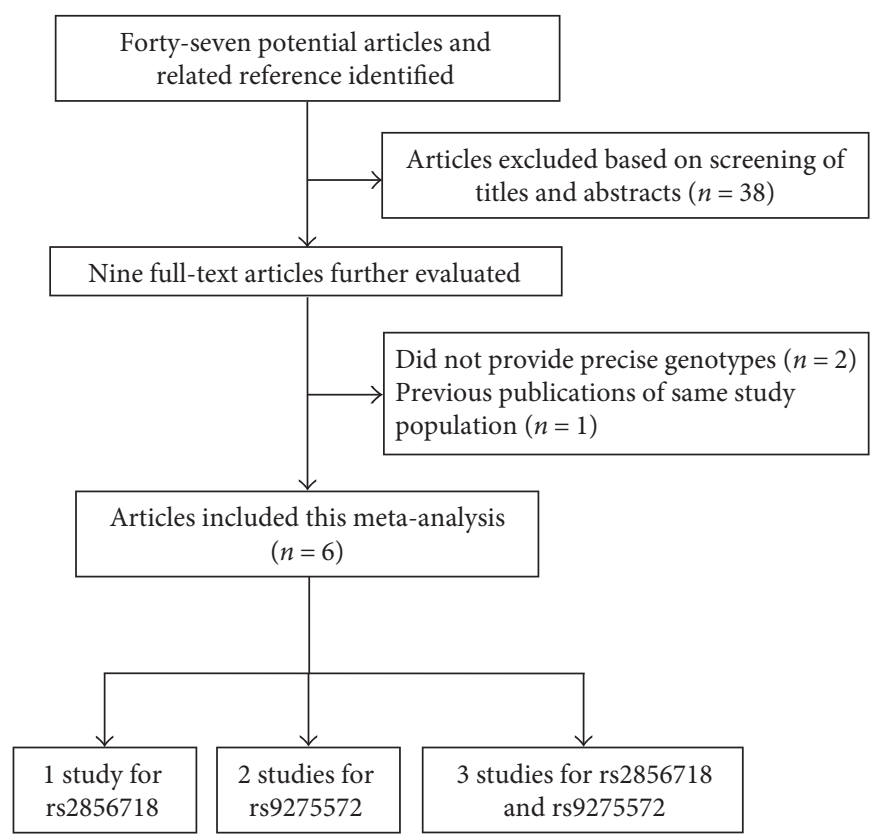

FIgURE 1: The flow charts of literature search and study selection.

polymorphism was significantly associated with $\mathrm{HCV}$ related HCC in Japanese population [14]. Chen et al.'s study found that HLA-DQ rs9275572 polymorphism may have a protective impact on HBV-related HCC [15].

Previous studies have assessed the association between HLA-DQ (rs2856718 and rs9275572) polymorphisms and $\mathrm{HBV}$-related HCC susceptibility, but the results of previous studies are inconsistent and inconclusive [13, 15-19]. Therefore, we performed a comprehensive meta-analysis to derive a more precise estimation of the relationship between HLA-DQ (rs2856718 and rs9275572) polymorphisms and HBV-related HCC risk. To the best of our knowledge, this is the first meta-analysis to analyze the association of the HLA-DQ (rs2856718 and rs9275572) polymorphisms with HBV-related HCC risk.

\section{Material and Methods}

2.1. Search Strategy. To identify relevant studies, we systematically searched PubMed, EMBASE, Google Scholar and China National Knowledge Infrastructure (CNKI) databases. The search strategy was based on a combination of "HLA-DQ," "hepatitis B virus," or "HBV"; "Hepatocellular carcinoma," "HCC," or "liver cancer"; "polymorphism" or "SNP"; and "rs2856718" or "rs9275572" (up to March 27, 2017). The languages of the reviewed articles were limited to English and Chinese. In addition, references of retrieved articles were also screened.

2.2. Inclusion and Exclusion Criteria. The following criteria were necessary for inclusion in the meta-analysis: (1) a case-control study that had investigated the genetic risk of HBV-related HCC in relation to HLA-DQ rs2856718 or rs9275572, (2) original papers containing independent data, (3) the study that provided the available genotype frequencies, (4) the study that provided sufficient information for calculating the pooled odds ratios (ORs) with 95\% confidence intervals (CIs), and (5) the genotype distribution of a control group that are consistent with the HardyWeinberg equilibrium (HWE).

Exclusion criteria were as follows: (1) case-only studies, (2) review articles, (3) repetitive reports, and (4) lack of genotype frequency data. In addition, if multiple studies had overlapping data, only the most recent version was used.

2.3. Data Extraction. The following data were independently extracted from each study by two authors (Jingzhu Lv and Tao $\mathrm{Xu}$ ): the first author's name, year of publication, country, genotyping method, number of cases and controls, genotype, and allele frequency. After extraction, data were reviewed and compared by the two independent investigators. Any disagreements were resolved by discussion with the third investigator.

2.4. Statistical Analysis. All statistical analyses were performed using Stata software version 12.0 (Stata Corporation, College Station, TX). The Hardy-Weinberg equilibrium test in the control group was undertaken using the $\chi^{2}$-test $(P<0.05$ was considered as significant disequilibrium in the control group). Odds ratios (ORs) with 95\% confidence intervals (95\% CIs) were used to estimate the strength of the association between HLA-DQ (rs2856718 and rs9275572) polymorphisms and HBV-related HCC. The pooled ORs were obtained from combination of single studies by homozygote comparison, heterozygote comparison, dominant and recessive models, and allele comparison, respectively. The significance of pooled ORs was assessed by the $\mathrm{Z}$ test, and $P_{\mathrm{Z}}<0.05$ was considered as the significance threshold. Based on the heterogeneity test, the pooled OR was calculated using the fixed $\left(P_{\mathrm{H}} \geq 0.05, I^{2} \leq 50 \%\right)$ or 


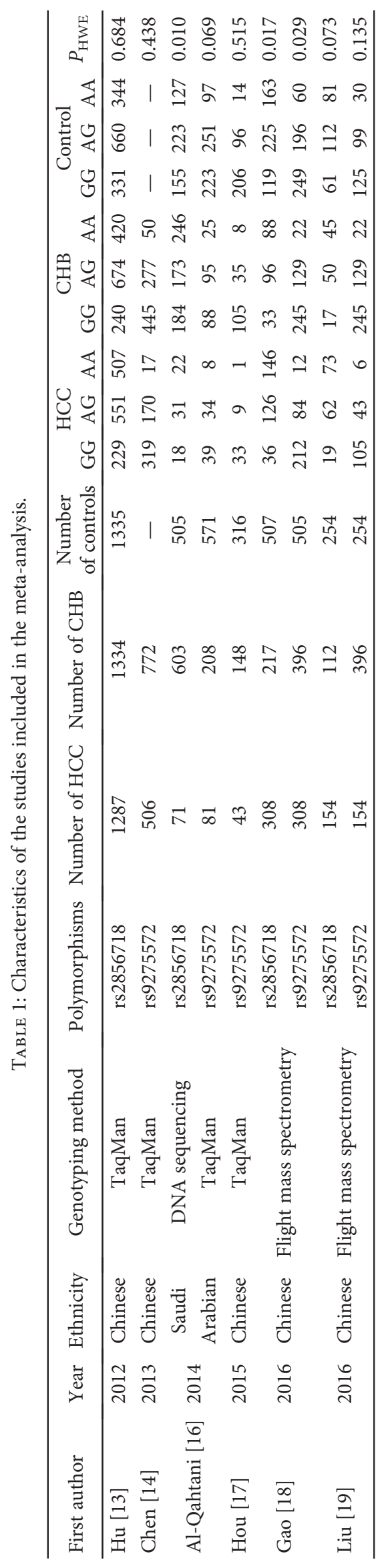


TABLE 2: Main results of the meta-analysis of the association between HLA-DQ (rs2856718) polymorphisms and the risk of HBV-related HCC.

\begin{tabular}{|c|c|c|c|c|c|c|c|}
\hline \multirow[t]{2}{*}{ Comparison } & \multirow[t]{2}{*}{ Subgroup } & \multirow[t]{2}{*}{ OR (95\% CI) } & \multirow[t]{2}{*}{ Model } & \multicolumn{2}{|c|}{$\begin{array}{l}\text { Heterogeneity } \\
\text { test }\end{array}$} & \multirow[t]{2}{*}{$P_{\mathrm{Z}}$} & \multirow[t]{2}{*}{$P_{\mathrm{E}}$} \\
\hline & & & & $I^{2}(\%)$ & $P_{\mathrm{H}}$ & & \\
\hline \multicolumn{8}{|l|}{ HCC versus control } \\
\hline \multirow{3}{*}{ Dominant model (AG + GG versus AA) } & Overall & $0.54(0.47-0.62)$ & $\mathrm{F}$ & 0 & 0.688 & $<0.001$ & 0.41 \\
\hline & Chinese & $0.53(0.46-0.61)$ & $\mathrm{F}$ & 0 & 0.990 & $<0.001$ & 0.06 \\
\hline & Saudi Arabian & $0.74(0.44-1.29)$ & * & * & * & 0.294 & * \\
\hline \multirow{3}{*}{ Recessive model (GG versus AG + AA) } & Overall & $0.60(0.51-0.70)$ & $\mathrm{F}$ & 42.7 & 0.155 & $<0.001$ & 0.51 \\
\hline & Chinese & $0.59(0.50-0.69)$ & $\mathrm{R}$ & 55.6 & 0.105 & $<0.001$ & 0.22 \\
\hline & Saudi Arabian & $0.77(0.43-1.35)$ & * & * & * & 0.36 & $*$ \\
\hline \multirow{3}{*}{ Homozygous model (GG versus AA) } & Overall & $0.44(0.37-0.53)$ & $\mathrm{F}$ & 23.2 & 0.271 & $<0.001$ & 0.82 \\
\hline & Chinese & $0.43(0.36-0.51)$ & $\mathrm{F}$ & 13.7 & 0.314 & $<0.001$ & 0.24 \\
\hline & Saudi Arabian & $0.67(0.34-1.30)$ & * & * & * & 0.24 & * \\
\hline \multirow{3}{*}{ Heterozygous model (AG versus AA) } & Overall & $0.59(0.52-0.68)$ & $\mathrm{F}$ & 0 & 0.704 & $<0.001$ & 0.09 \\
\hline & Chinese & $0.58(0.50-0.68)$ & $\mathrm{F}$ & 0 & 0.841 & $<0.001$ & 0.32 \\
\hline & Saudi Arabian & $0.80(0.45-1.45)$ & * & * & * & 0.46 & * \\
\hline \multirow{3}{*}{ Allele model ( $G$ versus A) } & Overall & $0.64(0.58-0.70)$ & $\mathrm{F}$ & 23.0 & 0.273 & $<0.001$ & 0.88 \\
\hline & Chinese & $0.63(0.57-0.69)$ & $\mathrm{F}$ & 9.8 & 0.330 & $<0.001$ & 0.22 \\
\hline & Saudi Arabian & $0.80(0.50-1.14)$ & * & * & * & 0.21 & * \\
\hline \multicolumn{8}{|l|}{ HCC versus $\mathrm{CHB}$} \\
\hline \multirow{3}{*}{ Dominant model (AG + GG versus AA) } & Overall & $0.83(0.63-1.10)$ & $\mathrm{R}$ & $60.4 \%$ & 0.056 & 0.194 & 0.308 \\
\hline & Chinese & $0.72(0.62-0.83)$ & $\mathrm{F}$ & 0 & 0.093 & $<0.001$ & 0.283 \\
\hline & Saudi Arabian & $1.53(0.90-2.60)$ & $*$ & * & * & 0.112 & $*$ \\
\hline \multirow{3}{*}{ Recessive model (GG versus $A G+A A)$} & Overall & $0.92(0.78-1.09)$ & $\mathrm{F}$ & 0 & 0.626 & 0.342 & 0.058 \\
\hline & Chinese & $0.94(0.78-1.12)$ & $\mathrm{F}$ & 0 & 0.511 & 0.485 & 0.251 \\
\hline & Saudi Arabian & $0.77(0.44-1.36)$ & $\mathrm{F}$ & 0 & * & 0.370 & $*$ \\
\hline \multirow{3}{*}{ Homozygous model (GG versus AA) } & Overall & $0.79(0.69-0.95)$ & $\mathrm{F}$ & 0 & 0.676 & 0.014 & 0.982 \\
\hline & Chinese & $0.78(0.63-0.93)$ & $\mathrm{F}$ & 0 & 0.796 & 0.008 & 0.271 \\
\hline & Saudi Arabian & $1.09(0.57-2.10)$ & $\mathrm{F}$ & 0 & $*$ & 0.787 & $*$ \\
\hline \multirow{3}{*}{ Heterozygous model (AG versus AA) } & Overall & $0.90(0.61-1.32)$ & $\mathrm{R}$ & 75.9 & 0.006 & 0.579 & 0.245 \\
\hline & Chinese & $0.70(0.60-0.81)$ & $\mathrm{F}$ & 0 & 0.719 & $<0.001$ & 0.281 \\
\hline & Saudi Arabian & $2.00(1.12-3.58)$ & $\mathrm{F}$ & 0 & $*$ & 0.019 & * \\
\hline \multirow{3}{*}{ Allele model (G versus A) } & Overall & $0.85(0.78-0.94)$ & $\mathrm{F}$ & 0 & 0.486 & 0.001 & 0.707 \\
\hline & Chinese & $0.84(0.76-0.92)$ & $\mathrm{F}$ & 0 & 0.887 & $<0.001$ & 0.229 \\
\hline & Saudi Arabian & $1.10(0.77-1.56)$ & $\mathrm{F}$ & 0 & $*$ & 0.599 & * \\
\hline
\end{tabular}

OR: odds ratio; CI: confidence interval; $P_{\mathrm{H}}: P$ value of heterogeneity test; $P_{\mathrm{Z}}: P$ value of $\mathrm{Z}$ test; $P_{\mathrm{E}}: P$ value of Egger's test; R: random effect model; F: fixed effect model. *Because there was only one study with this genotype of rs 2856718 , the value could not be calculated.

random $\left(P_{\mathrm{H}}<0.05, I^{2}>50 \%\right)$ effect models. Sensitivity analysis was carried out to identify the effect of data from each study on the pooled ORs. Finally, publication bias was assessed using Egger's test, and $P_{\mathrm{E}}<0.05$ was considered statistically significant.

\section{Results}

3.1. Characteristics of the Included Studies. A total of 47 potentially relevant articles published up to March 27, 2017 were systematically identified in PubMed, EMBASE, Google Scholar, and CNKI. The flow chart that summarized the literature review process and the specific reasons for any exclusion from the meta-analysis are shown in Figure 1. In the end, 6 articles that met the inclusion criteria were included in the meta-analysis of 1 study for HLA-DQ rs2856718, 2 studies for HLA-DQ rs9275572, and 3 studies for HLA-DQ rs2856718 and HLA-DQ rs9275572 [13, 15-19]. Characteristics of all eligible articles are shown in Table 1 . Of the 6 articles, 4 articles including 1820 cases, $2266 \mathrm{CHB}$, and 2601 controls evaluated the association between HLA-DQ rs2856718 and HBV-related HCC risk, while 5 studies evaluated the association between the HLA-DQ rs9275572 and HBV-related HCC risk (1092 cases, $1920 \mathrm{CHB}$, and 1646 controls).

3.2. Meta-Analysis of the Association between HLA-DQ rs2856718 and HBV-Related HCC Risk. The potential 


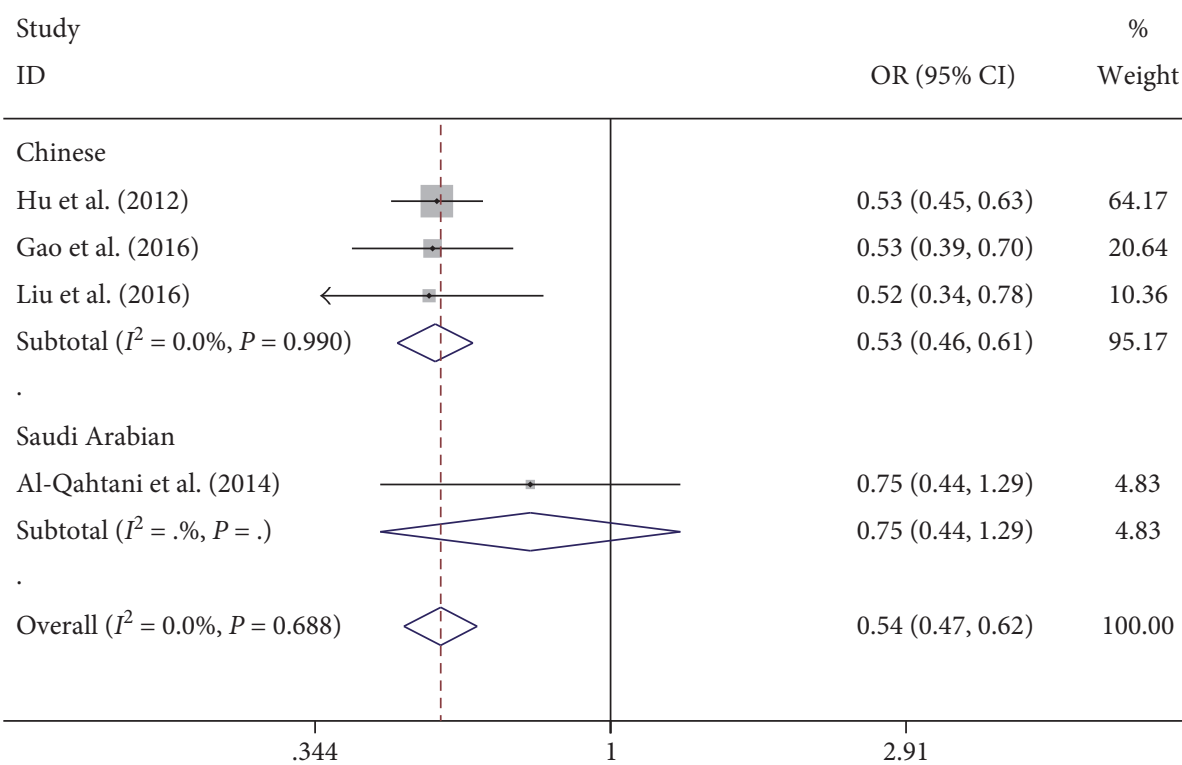

(a)

Study

ID

OR (95\% CI) Weight

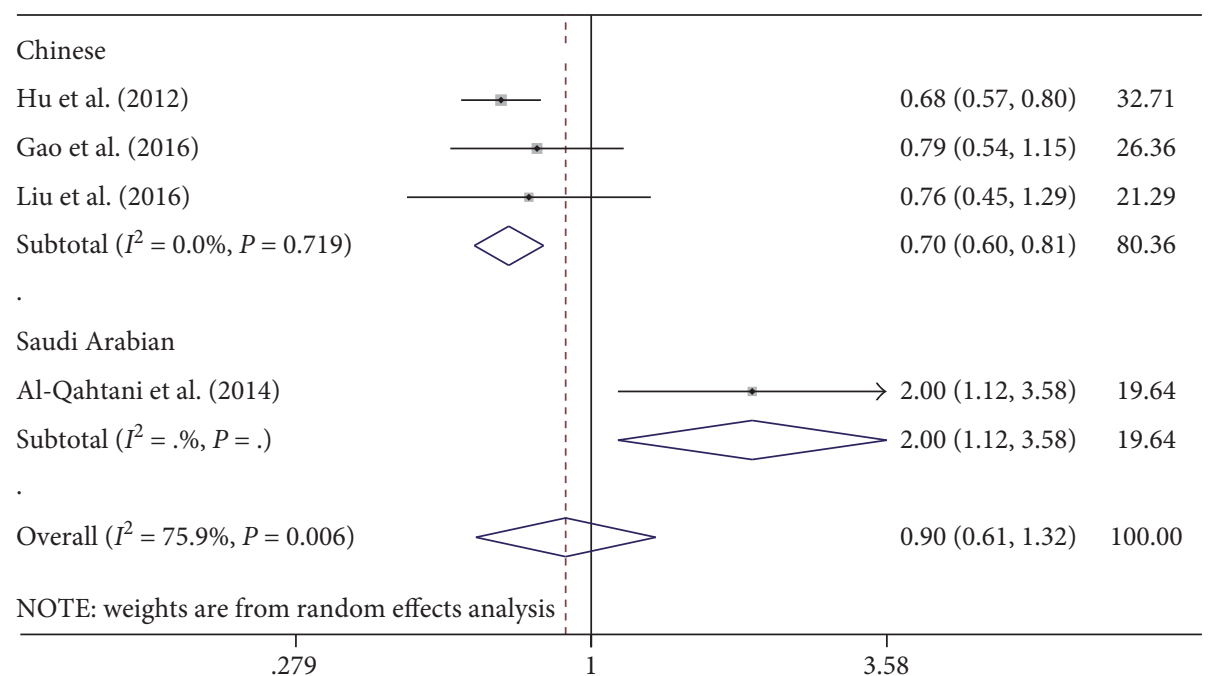

(b)

FIGURE 2: Forest plots for HLA-DQ rs2856718 polymorphism and the risk of HBV-related HCC. (a) Overall meta-analysis of the relationship between HLA-DQ rs2856718 polymorphism and HBV-related HCC (HCC versus control) risk in dominant model (AG + GG versus AA). (b) Overall meta-analysis of the relationship between HLA-DQ rs2856718 polymorphism and HBV-related HCC (HCC versus CHB) risk in heterozygous model (AG versus AA).

association of the HLA-DQ rs2856718 and HBV-related HCC was investigated in four articles; among which, three articles were in Chinese population and one in Saudi Arabian population. The meta-analysis of a possible association between the HLA-DQ rs2856718 and risk of HBV-related HCC is summarized in Table 2. The result of the controls and HBV-related HCC cases indicated a strong association of HLA-DQ rs2856718 with HBV-related HCC susceptibility. When the two populations were combined together, the overall OR for dominant model contrast (AG + GG versus
AA: $\left.\mathrm{OR}=0.54,95 \% \mathrm{CI}=0.47-0.62, P_{\mathrm{Z}}<0.001\right)$, recessive contrast ( $\mathrm{GG}$ versus $\mathrm{AG}+\mathrm{AA}$ : $\mathrm{OR}=0.60,95 \% \mathrm{CI}=0.51-$ $0.70, P_{\mathrm{Z}}<0.001$ ), homozygous contrast (GG versus AA: $\mathrm{OR}=0.44,95 \%$ CI: $\left.0.37-0.53, P_{\mathrm{Z}}<0.001\right)$, heterozygous contrast (AG versus AA: $\mathrm{OR}=0.59,95 \%$ CI: $0.52-0.68$, $\left.P_{\mathrm{Z}}<0.001\right)$, and allele contrast ( $\mathrm{G}$ versus $\mathrm{A}$ : $\mathrm{OR}=0.64,95 \%$ CI: $\left.0.58-0.70, P_{\mathrm{Z}}<0.001\right)$ was significantly associated with HBV-related HCC (Figure 2(a)). Additionally, this risk was more significant in the Chinese population, but there was no association between the HLA-DQ rs2856718 and HBV- 
TABLE 3: Main results of the meta-analysis of the association between HLA-DQ (rs9275572) polymorphisms and the risk of HBV-related HCC.

\begin{tabular}{|c|c|c|c|c|c|c|c|}
\hline \multirow[t]{2}{*}{ Comparison } & \multirow[t]{2}{*}{ Subgroup } & \multirow[t]{2}{*}{ OR $(95 \% \mathrm{CI})$} & \multirow[t]{2}{*}{ Model } & \multicolumn{2}{|c|}{$\begin{array}{c}\text { Heterogeneity } \\
\text { test }\end{array}$} & \multirow[t]{2}{*}{$P_{\mathrm{Z}}$} & \multirow[t]{2}{*}{$P_{\mathrm{E}}$} \\
\hline & & & & $I^{2}(\%)$ & $P_{\mathrm{H}}$ & & \\
\hline \multicolumn{8}{|l|}{ HCC versus control } \\
\hline \multirow{3}{*}{ Dominant model (AA + AG versus GG) } & Overall & $0.49(0.40-0.61)$ & $\mathrm{F}$ & 0 & 0.416 & $<0.001$ & 0.391 \\
\hline & Chinese & $0.46(0.36-0.57)$ & $\mathrm{F}$ & 0 & 0.825 & $<0.001$ & 0.168 \\
\hline & Saudi Arabian & $0.69(0.43-1.10)$ & * & * & $*$ & 0.120 & * \\
\hline \multirow{3}{*}{ Recessive model (AA versus AG + GG) } & Overall & $0.37(0.24-0.56)$ & $\mathrm{F}$ & 0 & 0.659 & $<0.001$ & 0.698 \\
\hline & Chinese & $0.31(0.19-0.52)$ & $\mathrm{F}$ & 0 & 0.885 & $<0.001$ & 0.216 \\
\hline & Saudi Arabian & $0.54(0.25-1.15)$ & * & * & * & 0.108 & $*$ \\
\hline \multirow{3}{*}{ Homozygous model (AA versus GG) } & Overall & $0.29(0.19-0.45)$ & $\mathrm{F}$ & 0 & 0.537 & $<0.001$ & 0.652 \\
\hline & Chinese & $0.25(0.15-0.41)$ & $\mathrm{F}$ & 0 & 0.842 & $<0.001$ & 0.208 \\
\hline & Saudi Arabian & $0.47(0.27-1.05)$ & * & $*$ & * & 0.065 & * \\
\hline \multirow{3}{*}{ Heterozygous model (AG versus GG) } & Overall & $0.56(0.45-0.69)$ & $\mathrm{F}$ & 0 & 0.525 & $<0.001$ & 0.491 \\
\hline & Chinese & $0.52(0.40-0.66)$ & $\mathrm{F}$ & 0 & 0.939 & $<0.001$ & 0.097 \\
\hline & Saudi Arabian & $0.77(0.47-1.27)$ & * & $*$ & * & 0.311 & * \\
\hline \multirow{3}{*}{ Allele model (A versus G) } & Overall & $0.52(0.44-0.62)$ & $\mathrm{F}$ & 0 & 0.280 & $<0.001$ & 0.501 \\
\hline & Chinese & $0.48(0.40-0.58)$ & $\mathrm{F}$ & 0 & 0.782 & $<0.001$ & 0.165 \\
\hline & Saudi Arabian & $0.70(0.49-1.00)$ & * & 21.8 & * & 0.048 & * \\
\hline \multicolumn{8}{|l|}{$\mathrm{HCC}$ versus $\mathrm{CHB}$} \\
\hline \multirow{3}{*}{ Dominant model (AA + AG versus GG) } & Overall & $0.75(0.64-0.88)$ & $\mathrm{F}$ & 0 & 0.832 & $<0.001$ & 0.674 \\
\hline & Chinese & $0.74(0.63-0.88)$ & $\mathrm{F}$ & 0 & 0.700 & 0.001 & 0.616 \\
\hline & Saudi Arabian & $0.79(0.47-1.32)$ & $*$ & $*$ & $*$ & 0.369 & $*$ \\
\hline \multirow{3}{*}{ Recessive model (AA versus AG + GG) } & Overall & $0.61(0.42-0.87)$ & $\mathrm{F}$ & 0 & 0.885 & 0.007 & 0.780 \\
\hline & Chinese & $0.57(0.38-0.85)$ & $\mathrm{F}$ & 0 & 0.882 & 0.006 & 0.877 \\
\hline & Saudi Arabian & $0.80(0.35-1.86)$ & $*$ & $*$ & $*$ & 0.608 & $*$ \\
\hline \multirow{3}{*}{ Homozygous model (AA versus GG) } & Overall & $0.55(0.38-0.80)$ & $\mathrm{F}$ & 0 & 0.934 & 0.002 & 0.766 \\
\hline & Chinese & $0.52(0.35-0.79)$ & $\mathrm{F}$ & 0 & 0.934 & 0.002 & 0.897 \\
\hline & Saudi Arabian & $0.72(0.30-1.74)$ & $*$ & $*$ & $*$ & 0.469 & $*$ \\
\hline \multirow{3}{*}{ Heterozygous model (AG versus GG) } & Overall & $0.78(0.66-0.93)$ & $\mathrm{F}$ & 0 & 0.727 & 0.005 & 0.651 \\
\hline & Chinese & $0.78(0.65-0.93)$ & $\mathrm{F}$ & 0 & 0.566 & 0.007 & 0.649 \\
\hline & Saudi Arabian & $0.81(0.47-1.39)$ & $*$ & $*$ & * & 0.441 & $*$ \\
\hline \multirow{3}{*}{ Allele model (A versus G) } & Overall & $0.76(0.67-0.87)$ & $\mathrm{F}$ & 0 & 0.916 & $<0.001$ & 0.750 \\
\hline & Chinese & $0.75(0.65-0.87)$ & $\mathrm{F}$ & 0 & 0.869 & $<0.001$ & 0.529 \\
\hline & Saudi Arabian & $0.83(0.57-1.23)$ & * & $*$ & * & 0.362 & * \\
\hline
\end{tabular}

OR: odds ratio; CI: confidence interval, $P_{\mathrm{H}}: P$ value of heterogeneity test; $P_{\mathrm{Z}}: P$ value of $\mathrm{Z}$ test; $P_{\mathrm{E}}: P$ value of Egger's test; R: random effect model; F: fixed effect model. * Because there was only one study with this genotype of rs 9275572 , the value could not be calculated.

related HCC susceptibility in Saudi Arabian population. When the controls were $\mathrm{CHB}$ patients, only GG versus AA and $G$ versus A of HLA-DQ rs2856718 were significantly associated with the risk of HBV-related HCC. However, subgroup analysis by ethnicity showed that HLA-DQ rs2856718 AG genotype had a significantly increased risk of $\mathrm{HBV}$ related HCC among Saudi Arabian population (AG versus AA: $O R=2.00,95 \%$ CI: $\left.1.12-3.58, P_{Z}=0.019\right)$ (Figure $2(b)$ ).

3.3. Meta-Analysis of the Association between HLA-DQ rs9275572 and HBV-Related HCC Risk. Five studies reported a potential association between HLA-DQ rs9275572 polymorphism and HBV-related HCC risk with evidence from 1092 cases and 3566 controls (the healthy group and CHB group). Table 3 shows the results for the association between HLA-DQ rs9275572 polymorphism and HBVrelated HCC risk. When the controls were the healthy group, the HLA-DQ rs9275572 polymorphism was associated with decreased $\mathrm{HBV}$-related HCC risk in all genetic models ( $\mathrm{AA}+\mathrm{AG}$ versus $\mathrm{GG}$ : $\mathrm{OR}=0.49$, 95\% CI: 0.40 $0.61, P_{Z}<0.001$; AA versus $\mathrm{AG}+\mathrm{GG}$ : $\mathrm{OR}=0.37,95 \% \mathrm{CI}$ : $0.24-0.56, P_{Z}<0.001$; AA versus $\mathrm{GG}$ : $\mathrm{OR}=0.29,95 \% \mathrm{CI}$ : $0.19-0.45, P_{Z}<0.001$; AG versus $G G$ : $\mathrm{OR}=0.56,95 \% \mathrm{CI}$ : $0.45-0.69, P_{\mathrm{Z}}<0.001$; and A versus $\mathrm{G}$ : $\mathrm{OR}=0.52,95 \% \mathrm{CI}$ : $\left.0.44-0.62, P_{Z}<0.001\right)$, particularly in Chinese population, but not among Saudi Arabian population (Figure 3(a)). Similar results were found when the controls were the $\mathrm{CHB}$ group $(\mathrm{AA}+\mathrm{AG}$ versus $\mathrm{GG}$ : $\mathrm{OR}=0.75,95 \% \mathrm{CI}$ : 


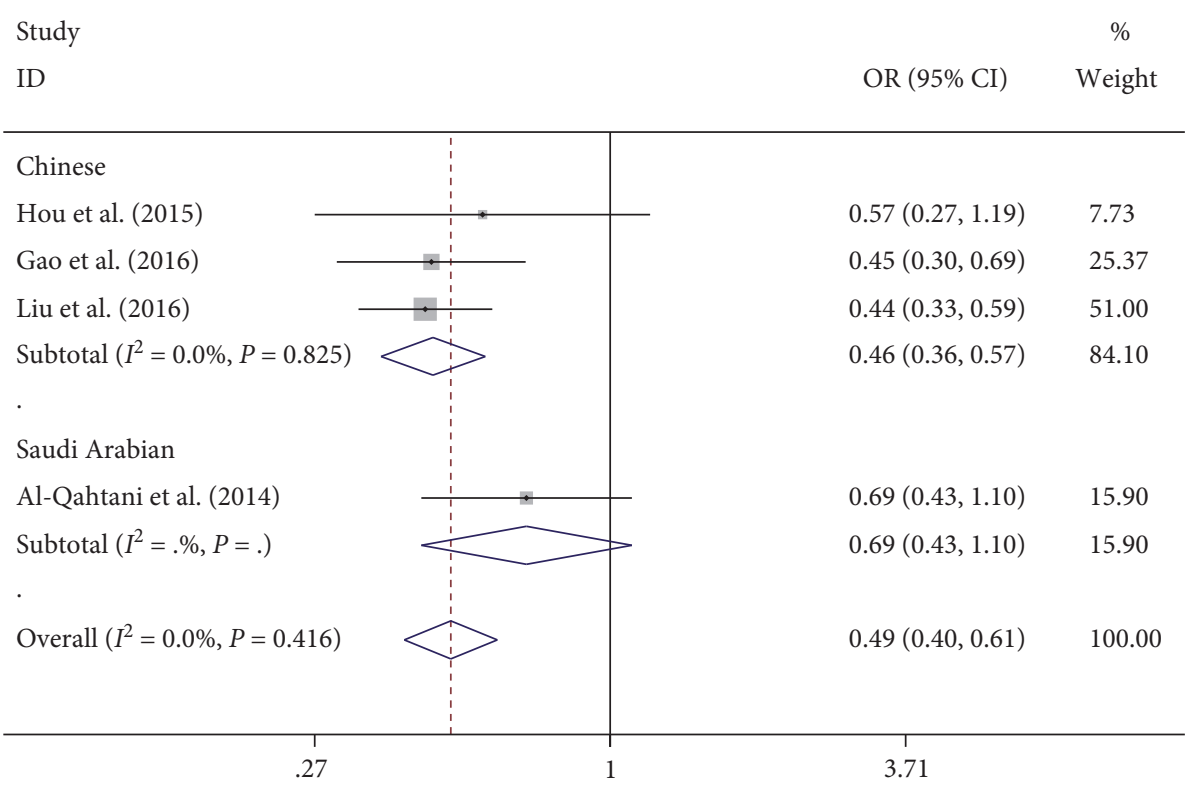

(a)

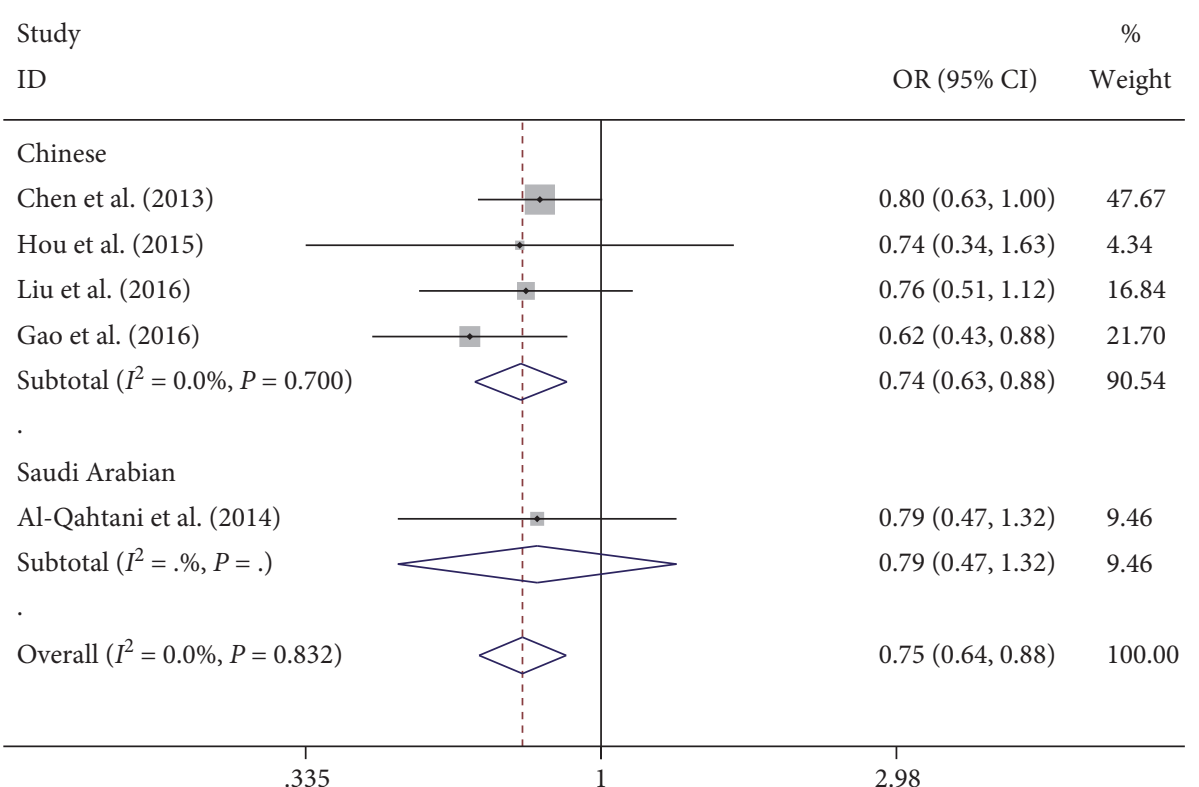

(b)

FIGURE 3: Forest plots for HLA-DQ rs9275572 polymorphism and the risk of HBV-related HCC. (a) Overall meta-analysis of the relationship between HLA-DQ rs9275572 polymorphism and HBV-related HCC (HCC versus control) risk in dominant model (AA + AG versus GG). (b) Overall meta-analysis of the relationship between HLA-DQ rs9275572 polymorphism and HBV-related HCC (HCC versus CHB) risk in dominant model (AA + AG versus GG).

0.64-0.88, $P_{Z}<0.001 ;$ AA versus $A G+G G: O R=0.61,95 \%$ CI: $0.42-0.87, P_{Z}=0.007$; AA versus $G G$ : $O R=0.55,95 \%$ CI: $0.38-0.80, P_{Z}=0.002$; $A G$ versus $G G$ : $O R=0.78,95 \%$ CI: $0.66-0.93, P_{Z}=0.005$; and A versus $G$ : $O R=0.76,95 \%$ CI: $0.67-0.87, P_{\mathrm{Z}}<0.001$ ) (Table 3 and Figure $3(\mathrm{~b})$ ).

3.4. Sensitivity Analysis. The sensitivity analysis was performed to assess the influence of an individual study on the overall OR, and the corresponding pooled ORs were not materially altered (Figures 4 and 5).
3.5. Publication Bias. Publication bias of the included articles was assessed using Egger's test. The results of Egger's test indicated that no evidence of publication bias was observed in HLA-DQ (rs2856718 and rs9275572) polymorphisms (Tables 2 and 3).

\section{Discussion}

HLA-DQs belong to HLA class II molecules, which are expressed as cell-surface glycoproteins that present viral 


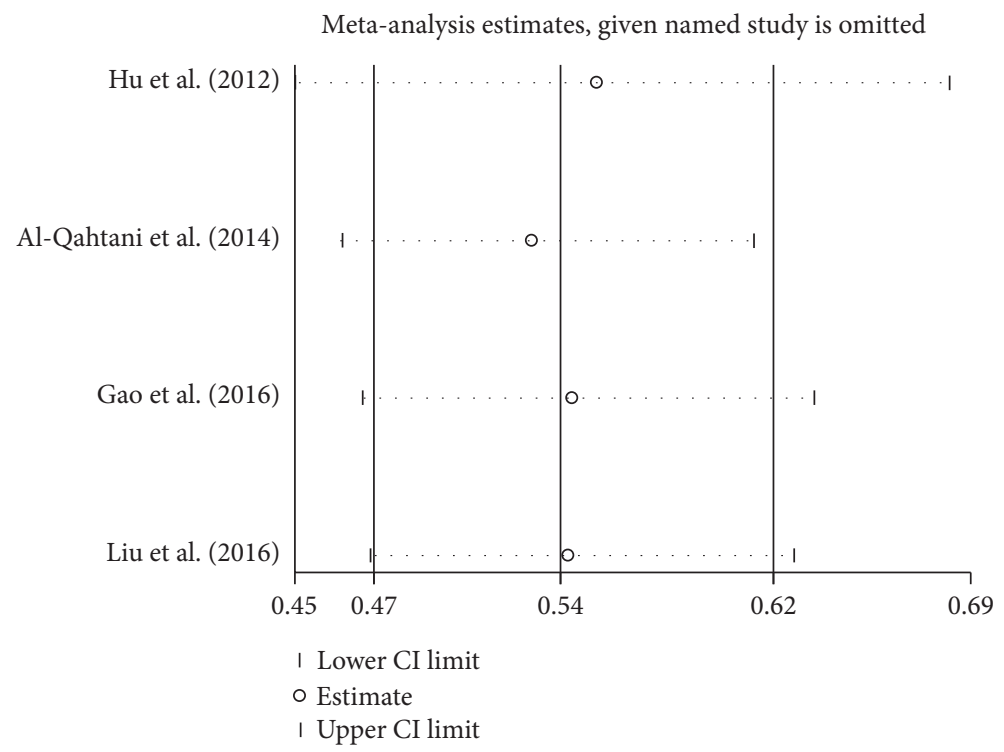

(a)

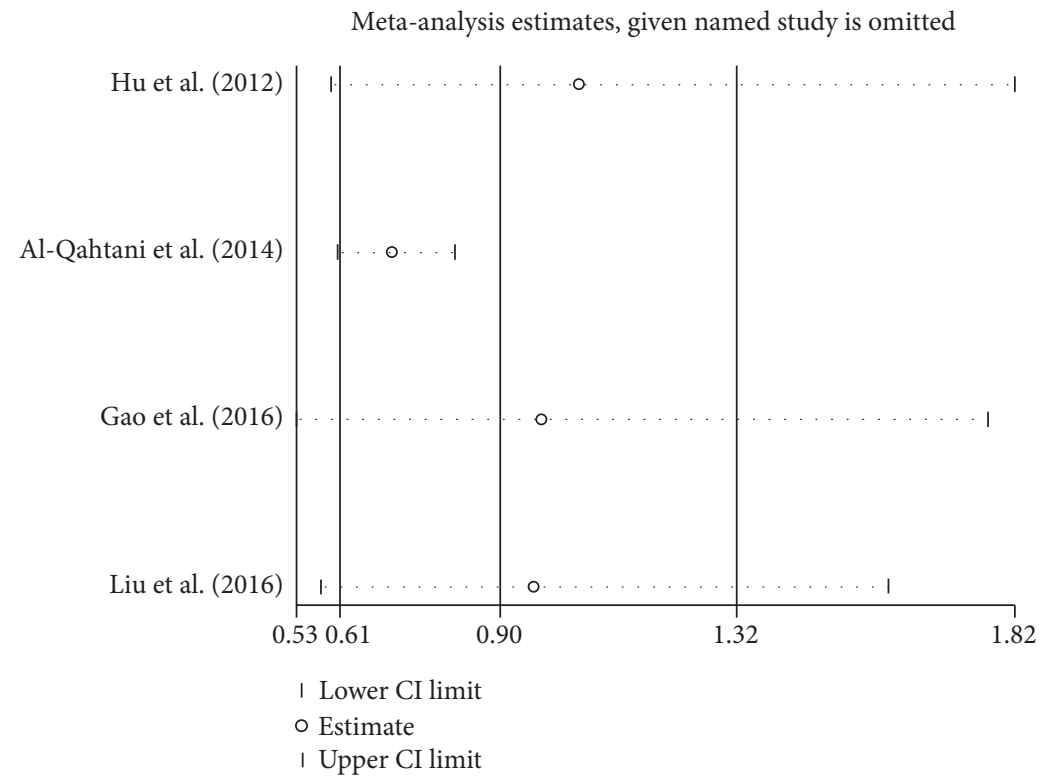

(b)

FIgURE 4: Sensitivity analysis of the pooled ORs and 95\% CIs for HLA-DQ rs2856718 polymorphism. (a) The sensitivity analysis results of rs 2856718 with HBV-related HCC (HCC versus control) in dominant model (AG + GG versus AA). (b) The sensitivity analysis results of rs2856718 with HBV-related HCC (HCC versus CHB) in heterozygous model (AG versus AA).

peptides to $\mathrm{CD} 4^{+} \mathrm{T}$ cells resulting in generating immunity against infection [20]. A study showed that the $\mathrm{CD} 4^{+} \mathrm{T}$ cells were significantly increased in tumor, ascites, and peripheral blood of patients with HCC and showed that HBV-specific and HLA class II-restricted $\mathrm{CD} 4^{+} \mathrm{T}$ cell responses may be related to HBV-related HCC development [21]. Recently, several studies have shown that genetic variations in HLA genes influence disease progression in HBV infection [22-25]. Accumulating evidence indicated the associations between HLA-DQ (rs2856718 and rs9275572) polymorphisms and HBV-related HCC, but the results are inconclusive or inconsistent. $\mathrm{Hu}$ et al.'s study showed that HLA-DQ rs2856718 polymorphism significantly decreased host HBV-related HCC risk in Southeast Han Chinese population [13]. Chen et al. confirmed that HLA-DQ rs9275572 polymorphism was significantly associated with HBV-related HCC risk in Chinese population [14], while Kumar et al. identified that it was associated with HCVrelated HCC in Japanese patients [15]. Gao et al.'s study suggested that HLA-DQ (rs2856718 and rs9275572) polymorphisms were the risk factor of HBV-related HCC [18]. Other's study found that HLA-DQ rs2856718G and rs9275572A might be protective factors for HBV infection, HBV natural clearance, and HBV-related HCC progress [19]. However, Hou et al. found that HLA-DQ rs9275572 polymorphism was significantly different from 


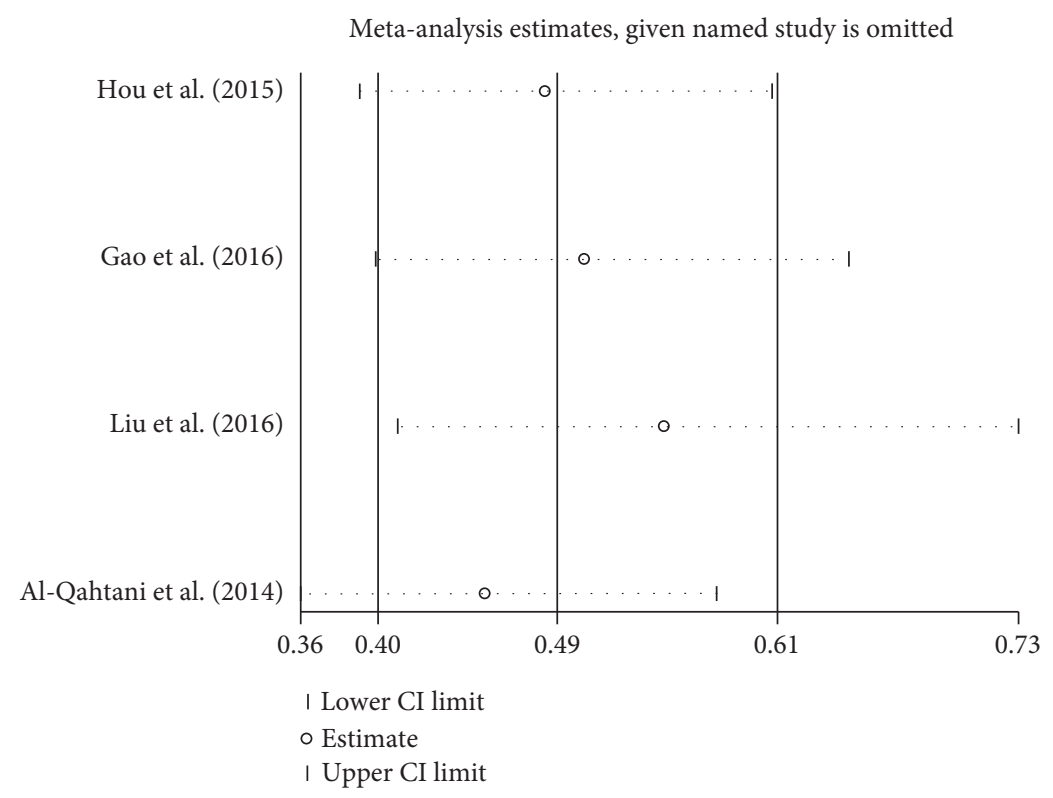

(a)

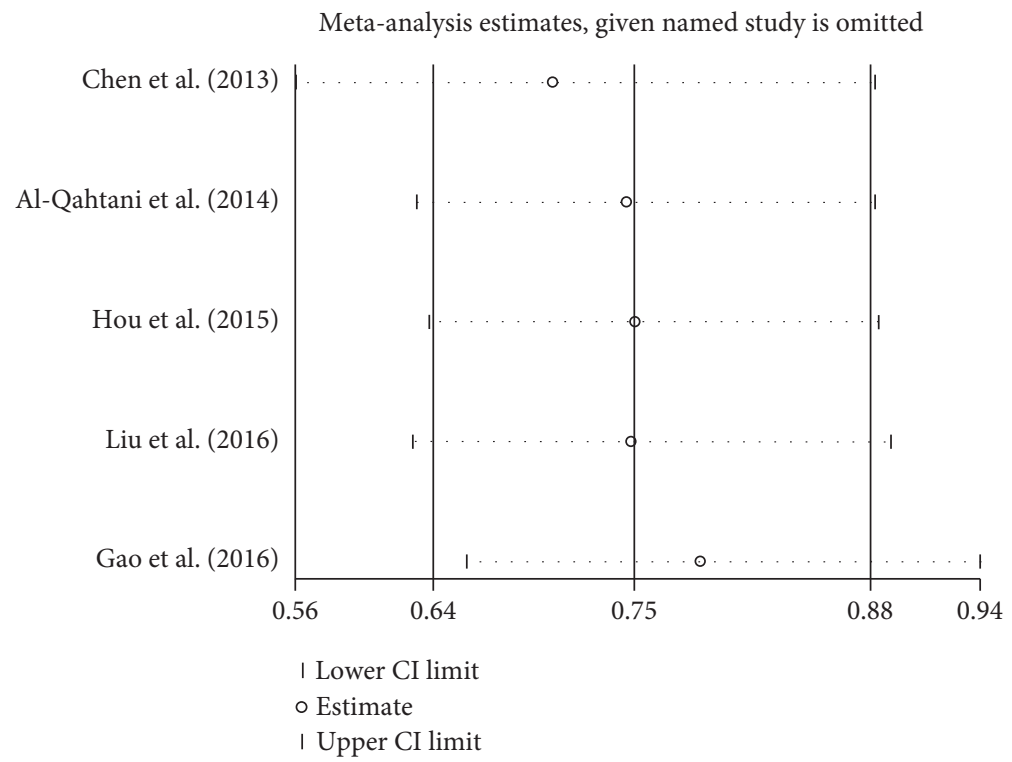

(b)

FIgURE 5: Sensitivity analysis of the pooled ORs and 95\% CIs for HLA-DQ rs9275572 polymorphism. (a) The sensitivity analysis results of rs9275572 with HBV-related HCC (HCC versus control) in dominant model (AA + AG versus GG). (b) The sensitivity analysis results of rs9275572 with HBV-related HCC (HCC versus CHB) in dominant model (AA + AG versus GG).

the HBV-related HCC [17]. Meanwhile, a study observed that there was no association between HLA-DQ (rs2856718 and rs9275572) polymorphisms and HBV-related HCC in Saudi Arabian patients [16].

In order to resolve this conflict, we conducted a metaanalysis on the association between HLA-DQ (rs2856718 and rs9275572) polymorphisms and HBV-related HCC. Meta-analysis has been recognized as an important tool to more precisely define the effect of selected genetic polymorphisms on the risk for disease and to identify potentially important sources of between-study heterogeneity. There are variations between human populations, so a SNP allele that is common in one geographical or ethnic group may be much rarer in another. So we also analyzed the distribution of HLA-DQ (rs2856718 and rs9275572) polymorphisms in different ethnic groups. Our results indicated that HLA-DQ (rs2856718 and rs9275572) polymorphisms were associated with the decreased risk of HBV-related HCC in Chinese population, but not in Saudi Arabian population. However, the sample size in Saudi Arabian population is too small to conclude a negative association. The comparison between Chinese population and Saudi Arabian population may be 
imbalanced as a result of the sample size. Therefore, the findings of this study should be validated in the future through a population-based study.

As a meta-analysis of observational studies, there are some limitations. Firstly, we did not have original data for all studies to adjust estimates and perform a more precise analysis, including gender, age, drinking, smoking, lifestyle, body mass index, and so on. Secondly, the number of published studies was not sufficiently large for a comprehensive analysis. Thirdly, the interaction of gene-gene and of geneenvironment has not been evaluated owing to the absence of original data. Therefore, more studies are needed to get more reliable results.

In conclusion, the current meta-analysis suggested that HLA-DQ (rs2856718 and rs9275572) polymorphisms were associated with HBV-related HCC risk among Chinese population. Taken together, our study suggested that HLA-DQ loci are candidate susceptibility regions that have some marker SNPs (rs2856718 and rs9275572) for HBV-related HCC in Chinese population.

\section{Conflicts of Interest}

The authors declare that they have no conflicts of interest.

\section{Acknowledgments}

This work was supported by Anhui Provincial Natural Science Research Project of University (KJ2013A188, KJ2014A164, and KJ2016A472), International Science and Technology Cooperation Project-Key Research and Development Program of Anhui Province (1604b0602026), and National Natural Science Foundation of China (81570011).

\section{References}

[1] R. L. Siegel, K. D. Miller, and A. Jemal, "Cancer statistics, 2015,” CA: A Cancer Journal for Clinicians, vol. 65, no. 1, pp. 5-29, 2015.

[2] J. Ferlay, D. M. Parkin, M. P. Curado et al., Cancer Incidence in five Continents, Volumes I to X: IARC CANCER Base No. 10, 2014, http://ci5.iarc.fr.

[3] K. A. McGlynn, J. L. Petrick, and W. T. London, “Global epidemiology of hepatocellular carcinoma: an emphasis on demographic and regional variability," Clinics in Liver Disease, vol. 19, no. 2, pp. 223-238, 2015.

[4] K. A. McGlynn and W. T. London, "The global epidemiology of hepatocellular carcinoma: present and future," Clinics in Liver Disease, vol. 15, no. 2, pp. 223-243, 2011.

[5] D. M. Parkin, F. Bray, J. Ferlay, and P. Pisani, "Global cancer statistics, 2002," CA: A Cancer Journal for Clinicians, vol. 55, pp. 74-108, 2005.

[6] V. A. McCormack and P. Boffetta, “Today's lifestyles, tomorrow's cancers: trends in lifestyle risk factors for cancer in low- and middle-income countries," Annals of Oncology, vol. 22, no. 11, pp. 2349-2357, 2011.

[7] J. D. Yang and L. R. Roberts, "Hepatocellular carcinoma: a global view," Nature Reviews Gastroenterology \& Hepatology, vol. 7, no. 8, pp. 448-458, 2010.
[8] M. J. Bouchard and S. Navas-Martin, "Hepatitis B and C virus hepatocarcinogenesis: lessons learned and future challenges," Cancer Letters, vol. 305, no. 2, pp. 123-143, 2011.

[9] Y. Zheng, J. Huang, M. Zhan et al., "Genetic variants in the KDR gene is associated with the prognosis of transarterial chemoembolization treated hepatocellular carcinoma," Tumor Biology, vol. 35, no. 11, pp. 11473-11481, 2014.

[10] K. Ye, S. Chang, J. Li, X. Li, Y. Zhou, and Z. Wang, “A functional and protein-protein interaction analysis of neuroepithelial cell transforming gene 1 in hepatocellular carcinoma," Tumor Biology, vol. 35, no. 11, pp. 11219-11227, 2014.

[11] Y. Lu, Z. Wu, Q. Peng et al., "Role of IL-4 gene polymorphisms in HBV-related hepatocellular carcinoma in a Chinese population," PloS One, vol. 9, no. 10, article e110061, 2014.

[12] R. Singh, R. Kaul, A. Kaul, and K. Khan, "A comparative review of HLA associations with hepatitis B and C viral infections across global populations," World Journal of Gastroenterology, vol. 13, no. 12, pp. 1770-1787, 2007.

[13] L. Hu, X. Zhai, J. Liu et al., "Genetic variants in human leukocyte antigen/DP-DQ influence both hepatitis B virus clearance and hepatocellular carcinoma development," Hepatology, vol. 55, no. 5, pp. 1426-1431, 2012.

[14] K. Chen, W. Shi, Z. Xin et al., "Replication of genome wide association studies on hepatocellular carcinoma susceptibility loci in a Chinese population," PloS One, vol. 8, no. 10, article e77315, 2013.

[15] V. Kumar, N. Kato, Y. Urabe et al., "Genome-wide association study identifies a susceptibility locus for HCV-induced hepatocellular carcinoma," Nature Genetics, vol. 43, no. 5, pp. 455-458, 2011.

[16] A. A. Al-Qahtani, M. R. Al-Anazi, A. A. Abdo et al., "Association between HLA variations and chronic hepatitis B virus infection in Saudi Arabian patients," PloS One, vol. 9, no. 1, article e80445, 2014.

[17] S. H. Hou, J. Hu, Y. Zhang, Q. L. Li, and J. J. Guo, "Effects of interaction between genetic variants in human leukocyte antigen DQ and granulysin genes in Chinese Han subjects infected with hepatitis B virus," Microbiology and Immunology, vol. 59, no. 4, pp. 209-218, 2015.

[18] X. Gao, W. Liu, X. Zhang et al., "Genetic polymorphism of HLA-DQ confers susceptibility to hepatitis B virus-related hepatocellular carcinoma: a case-control study in Han population in China," Tumor Biology, vol. 37, no. 9, pp. 12103-12111, 2016.

[19] W. X. Liu, X. L. Zhang, X. Gao, L. Yang, B. Li, and D. Liu, "Association between HLA-DQ gene polymorphisms and different outcomes of hepatitis B virus infection," Chinese Journal of Epidemiology, vol. 37, no. 3, pp. 384-388, 2016, (Chinese).

[20] G. Diaz, M. Amicosante, D. Jaraquemada et al., "Functional analysis of HLA-DP polymorphism: a crucial role for DP beta residues $9,11,35,55,56,69$, and $84-87$ in $\mathrm{T}$ cell allorecognition and peptide binding," International Immunology, vol. 15, no. 5, pp. 565-576, 2003.

[21] L. A. Ormandy, T. Hillemann, H. Wedemeyer, M. P. Manns, T. F. Greten, and F. Korangy, "Increased populations of regulatory $\mathrm{T}$ cells in peripheral blood of patients with hepatocellular carcinoma," Cancer Research, vol. 65, no. 6, pp. 2457-2464, 2005.

[22] L. Wang, Z. Q. Zou, and K. Wang, "Clinical relevance of HLA gene variants in HBV infection," Journal of Immunology Research, vol. 2016, Article ID 9069375, 7 pages, 2016. 
[23] S. W. Chang, C. S. Fann, W. H. Su et al., "A genome-wide association study on chronic HBV infection and its clinical progression in male Han-Taiwanese," PloS One, vol. 9, no. 6, article e99724, 2014.

[24] S. Li, J. Qian, Y. Yang et al., "GWAS identifies novel susceptibility loci on 6p21.32 and 21q21.3 for hepatocellular carcinoma in chronic hepatitis B virus carriers," PLoS Genetics, vol. 8, no. 7, article e1002791, 2012.

[25] D. K. Jiang, J. Sun, G. Cao et al., "Genetic variants in STAT4 and HLA-DQ genes confer risk of hepatitis B virus-related hepatocellular carcinoma," Nature Genetics, vol. 45, no. 1, pp. 72-75, 2013. 


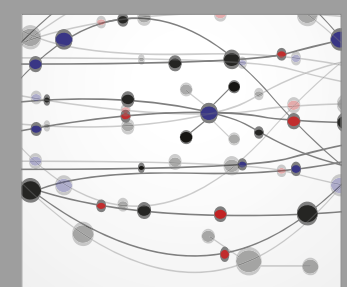

The Scientific World Journal
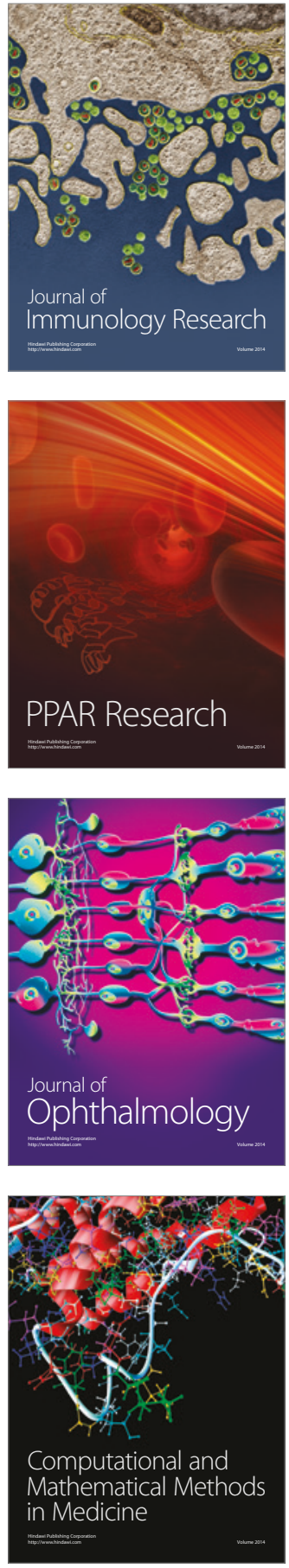

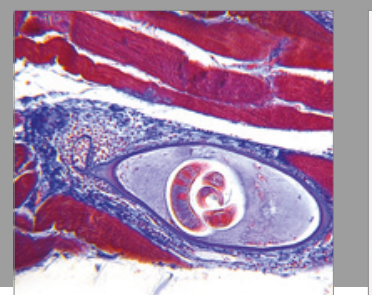

Gastroenterology Research and Practice
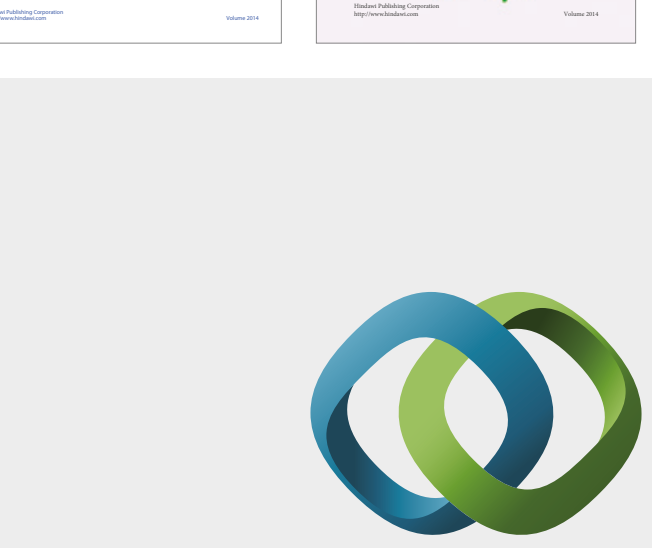

\section{Hindawi}

Submit your manuscripts at

https://www.hindawi.com
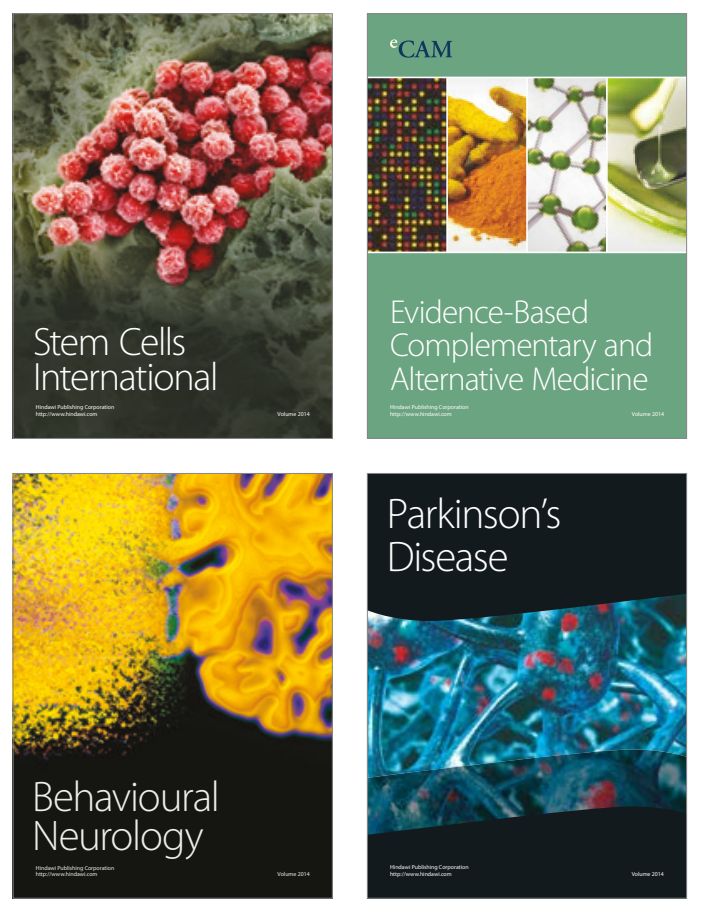
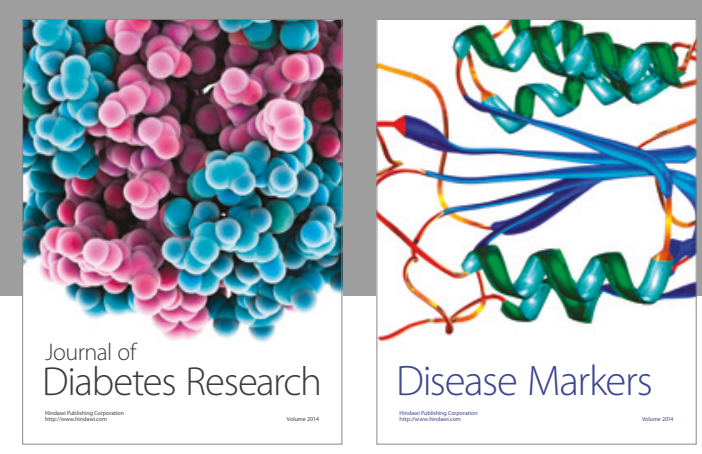

Disease Markers
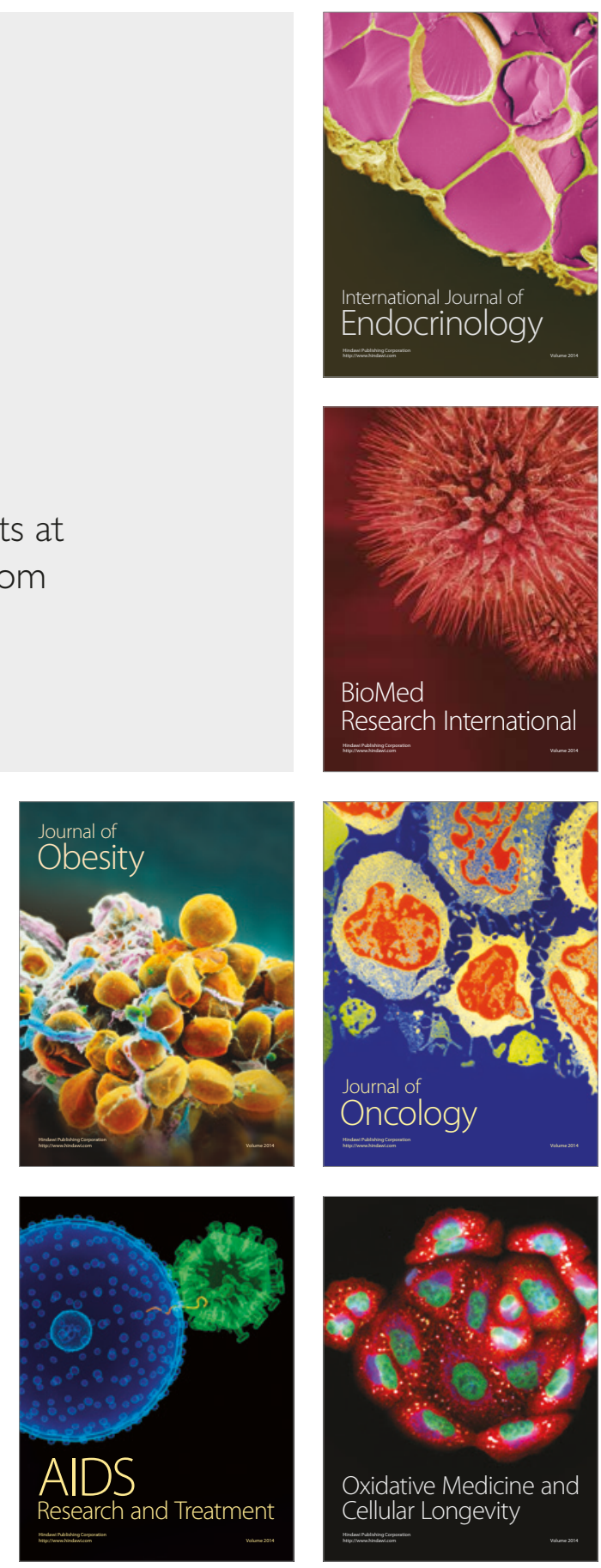